\title{
INVARIANT SETS AND NORMAL SUBGROUPOIDS OF UNIVERSAL ÉTALE GROUPOIDS INDUCED BY CONGRUENCES OF INVERSE SEMIGROUPS
}

\author{
FUYUTA KOMURA (D)
}

(Received 12 June 2020; accepted 21 January 2021; first published online 13 April 2021)

\author{
Communicated by Aidan Sims
}

\begin{abstract}
For a given inverse semigroup, one can associate an étale groupoid which is called the universal groupoid. Our motivation is studying the relation between inverse semigroups and associated étale groupoids. In this paper, we focus on congruences of inverse semigroups, which is a fundamental concept for considering quotients of inverse semigroups. We prove that a congruence of an inverse semigroup induces a closed invariant set and a normal subgroupoid of the universal groupoid. Then we show that the universal groupoid associated to a quotient inverse semigroup is described by the restriction and quotient of the original universal groupoid. Finally we compute invariant sets and normal subgroupoids induced by special congruences including abelianization.
\end{abstract}

2020 Mathematics subject classification: primary 20M18; secondary 22A22, 46L05.

Keywords and phrases: $\mathrm{C}^{*}$-algebras, étale groupoids.

\section{Introduction}

The relation among inverse semigroups, étale groupoids and $\mathrm{C}^{*}$-algebras has been revealed by many researchers. Paterson associated the universal groupoid to an inverse semigroup in [4]. He proved that the $C^{*}$-algebras associated to universal groupoids are isomorphic to inverse semigroup $C^{*}$-algebras. Furthermore, Paterson showed that the universal groupoid has a universal property about ample actions on totally disconnected spaces (also see [9]). In this paper we investigate a relation between inverse semigroups and universal groupoids. In particular, we focus on congruences of inverse semigroups, which is a fundamental concept for considering quotient inverse semigroups. Indeed, congruences of inverse semigroups induce closed invariant subsets and normal subgroupoids of universal groupoids. Our main theorem is that

This work was supported by JSPS KAKENHI $20 J 10088$.

(C) Australian Mathematical Publishing Association Inc. 2021. This is an Open Access article, distributed under the terms of the Creative Commons Attribution licence (http://creativecommons.org/ licenses/by/4.0/), which permits unrestricted re-use, distribution, and reproduction in any medium, provided the original work is properly cited. 
the universal groupoid of a quotient inverse semigroup is isomorphic to the restriction and quotient of the original universal groupoid (Theorem 4.3).

This paper is organized as follows. In Section 1, we review the notions of inverse semigroups and étale groupoids. In Section 2, we deal with some least congruences, although some statements may be well known for specialists. We also show that there is a one-to-one correspondence between normal congruences of semilattices and some invariant subsets (Corollary 3.6). In Section 3, we prove that the universal groupoid of a quotient inverse semigroup is isomorphic to the restriction and quotient of the original universal groupoid. Then we deal with some special congruences. In Section 4, we apply propositions and theorems obtained in Sections 2 and 3. We prove that the number of fixed points of a Boolean action is less than or equal to the number of certain semigroup homomorphisms (Corollary 5.17).

\section{Preliminaries}

2.1. Inverse semigroups. We recall basic facts about inverse semigroups. See [3, 4] for more details. An inverse semigroup $S$ is a semigroup where for every $s \in S$ there exists a unique $s^{*} \in S$ such that $s=s s^{*} s$ and $s^{*}=s^{*} s s^{*}$. We denote the set of all idempotents in $S$ by $E(S):=\left\{e \in S \mid e^{2}=e\right\}$. A zero element is a unique element $0 \in S$ such that $0 s=s 0=0$ holds for all $s \in S$. A unit is a unique element $1 \in S$ such that $1 s=s 1=s$ holds for all $s \in S$. An inverse semigroup does not necessarily have a zero element or a unit. It is known that $E(S)$ is a commutative subsemigroup of $S$. A subsemigroup of $S$ is a subset of $S$ that is closed under the product and inverse of $S$. A subsemigroup $N$ of $S$ is said to be normal if $E(S) \subset N$ and $s n s^{*} \in N$ holds for all $s \in S$ and $n \in N$. An order on $E(S)$ is defined by $e \leq f$ if $e f=e$.

An equivalence relation $v$ on $S$ is called a congruence if $(s, t) \in v$ implies (as,at), $(s a, t a) \in v$ for all $s, t, a \in S$. The quotient set $S / v$ becomes an inverse semigroup such that the quotient map $q: S \rightarrow S / v$ is a semigroup homomorphism. A congruence $\rho$ on $E(S)$ is said to be normal if $(e, f) \in \rho$ implies $\left(\right.$ ses $\left.^{*}, s f s^{*}\right) \in \rho$ for all $e, f \in E(S)$ and $s \in S$. One of the typical examples of normal congruences is $E(S) \times E(S)$. Assume that $\rho$ is a normal congruence on $E(S)$. Define an equivalence relation $v_{\rho, \min }$ on $S$ by declaring that $(s, t) \in v_{\rho \text {, min }}$ if $\left(s^{*} s, t^{*} t\right) \in \rho$ and $s e=t e$ holds for some $e \in E(S)$ with $\left(e, s^{*} s\right) \in \rho$. Then $v_{\rho \text {, min }}$ is the minimum congruence on $S$ such that its restriction to $E(S)$ coincides with $\rho$. One can see that $v_{E(S) \times E(S) \text {,min }}$ is the least congruence such that the quotient inverse semigroup becomes a group. We call $S / v_{E(S) \times E(S) \text {,min }}$ the maximal group image of $S$.

An inverse semigroup $S$ is said to be Clifford if $s^{*} s=s s^{*}$ holds for all $s \in S$. A congruence $v$ on $S$ is said to be Clifford if $S / v$ is Clifford.

2.2. Étale groupoids. We recall the notion of étale groupoids. See [7, 8] for more details. 
A groupoid is a set $G$ together with a distinguished subset $G^{(0)} \subset G$, source and range maps $d, r: G \rightarrow G^{(0)}$ and a multiplication

$$
G^{(2)}:=\{(\alpha, \beta) \in G \times G \mid d(\alpha)=r(\beta)\} \ni(\alpha, \beta) \mapsto \alpha \beta \in G
$$

such that:

(1) for all $x \in G^{(0)}, d(x)=x$ and $r(x)=x$ hold;

(2) for all $\alpha \in G, \alpha d(\alpha)=r(\alpha) \alpha=\alpha$ holds;

(3) for all $(\alpha, \beta) \in G^{(2)}, d(\alpha \beta)=d(\beta)$ and $r(\alpha \beta)=r(\alpha)$ hold;

(4) if $(\alpha, \beta),(\beta, \gamma) \in G^{(2)}$, we have $(\alpha \beta) \gamma=\alpha(\beta \gamma)$;

(5) for every $\gamma \in G$, there exists $\gamma^{\prime} \in G$ that satisfies $\left(\gamma^{\prime}, \gamma\right),\left(\gamma, \gamma^{\prime}\right) \in G^{(2)}$ and $d(\gamma)=$ $\gamma^{\prime} \gamma$ and $r(\gamma)=\gamma \gamma^{\prime}$.

Since the element $\gamma^{\prime}$ in (5) is uniquely determined by $\gamma, \gamma^{\prime}$ is called the inverse of $\gamma$ and denoted by $\gamma^{-1}$. We call $G^{(0)}$ the unit space of $G$. A subgroupoid of $G$ is a subset of $G$ that is closed under inversion and multiplication. For $U \subset G^{(0)}$, we define $G_{U}:=$ $d^{-1}(U)$ and $G^{U}:=r^{-1}(U)$. We define also $G_{x}:=G_{\{x\}}$ and $G^{x}:=G^{\{x\}}$ for $x \in G^{(0)}$. The isotropy bundle of $G$ is denoted by $\operatorname{Iso}(G):=\{\gamma \in G \mid d(\gamma)=r(\gamma)\}$. A subset $F \subset G^{(0)}$ is said to be invariant if $d(\alpha) \in F$ implies $r(\alpha) \in F$ for all $\alpha \in G$. We say that $x \in G^{(0)}$ is a fixed point if $\{x\} \subset G^{(0)}$ is invariant. If $G$ satisfies $G=\operatorname{Iso}(G), G$ is called a group bundle over $G^{(0)}$. A group bundle $G$ is said to be abelian if $G_{x}$ is an abelian group for all $x \in G^{(0)}$.

A topological groupoid is a groupoid equipped with a topology where the multiplication and the inverse are continuous. A topological groupoid is said to be étale if the source map is a local homeomorphism. Note that the range map of an étale groupoid is also a local homeomorphism. The next proposition easily follows from the definition of étale groupoids.

Proposition 2.1. Let $G$ and $H$ be étale groupoids. A groupoid homomorphism $\Phi: G \rightarrow H$ is a local homeomorphism if and only if $\left.\Phi\right|_{G^{(0)}}: G^{(0)} \rightarrow H^{(0)}$ is a local homeomorphism.

2.2.1. Quotient étale groupoids. We recall the notion of quotient étale groupoids. See [1, Section 3] for more details. Let $G$ be an étale groupoid. We say that a subgroupoid $H \subset G$ is normal if:

- $G^{(0)} \subset H \subset \operatorname{Iso}(G)$ holds; and

- for all $\alpha \in G, \alpha H \alpha^{-1} \subset H$ holds.

For a normal subgroupoid $H \subset G$, define an equivalence relation $\sim$ on $G$ by declaring that $\alpha \sim \beta$ if $d(\alpha)=d(\beta)$ and $\alpha \beta^{-1} \in H$ hold. Then $G / H:=G / \sim$ becomes a groupoid such that the quotient map is a groupoid homomorphism. If $H$ is open in $G$, then $G / H$ is an étale groupoid with respect to the quotient topology. Moreover, the quotient map is a local homeomorphism (see [1, Section 3.1] for these facts).

We have the fundamental homomorphism theorem. The proof is left to the reader. 
Proposition 2.2. Let $G$ and $H$ be étale groupoids and $\Phi: G \rightarrow H$ be a continuous groupoid homomorphism that is a local homeomorphism. Assume that $\Phi$ is injective on $G^{(0)}$. Then $\operatorname{ker} \Phi:=\Phi^{-1}\left(H^{(0)}\right)$ is an open normal subgroupoid of $G$. Moreover, there exists an isomorphism $\widetilde{\Phi}: G / \operatorname{ker} \Phi \rightarrow \Phi(G)$ that makes the following diagram commutative:

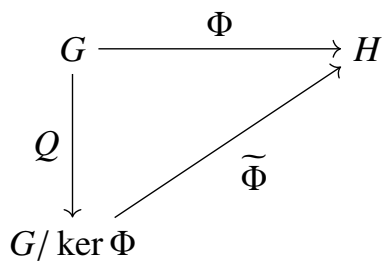

where $Q: G \rightarrow G / \operatorname{ker} \Phi$ denotes the quotient map.

For an étale groupoid $G$, the author of [1] constructed the étale abelian group bundle $G^{\mathrm{ab}}$. We briefly recall the definition of $G^{\mathrm{ab}}$.

We define $G_{\text {fix }}:=G_{F}$, where $F \subset G^{(0)}$ denotes the set of all fixed points. Then $G_{\text {fix }}$ is a group bundle. Define $\left[G_{\mathrm{fix}}, G_{\mathrm{fix}}\right]:=\bigcup_{x \in F}\left[\left(G_{\mathrm{fix}}\right)_{x},\left(G_{\mathrm{fix}}\right)_{x}\right]$, where $\left[\left(G_{\mathrm{fix}}\right)_{x},\left(G_{\mathrm{fix}}\right)_{x}\right]$ denotes the commutator subgroup of $\left(G_{\mathrm{fix}}\right)_{x}$. Then $\left[G_{\mathrm{fix}}, G_{\mathrm{fix}}\right]$ is an open normal subgroupoid of $G_{\text {fix }}$ [1, Proposition 4.9]. Now we define $G^{\mathrm{ab}}:=G_{\mathrm{fix}} /\left[G_{\mathrm{fix}}, G_{\mathrm{fix}}\right]$.

The reason why we consider $G^{\mathrm{ab}}$ is that the next theorem holds. We denote the universal groupoid $C^{*}$-algebra of $G$ by $C^{*}(G)$. See [4] for the definition of the universal groupoid $\mathrm{C}^{*}$-algebras.

THEOREM 2.3 [1, Theorem 4.12]. Let $G$ be an étale groupoid such that $G^{(0)}$ is a locally compact Hausdorff space with respect to the relative topology of $G$. Then $C^{*}\left(G^{\mathrm{ab}}\right)$ is the abelianization of $C^{*}(G)$ as a $C^{*}$-algebra.

2.3. Étale groupoids associated to inverse semigroup actions. Let $X$ be a topological space. We denote by $I_{X}$ the inverse semigroup of homeomorphisms between open sets in $X$. An action $\alpha: S \curvearrowright X$ is a semigroup homomorphism $S \ni s \mapsto \alpha_{s} \in I_{X}$. For $e \in E(S)$, we denote the domain of $\beta_{e}$ by $D_{e}^{\alpha}$. Then $\alpha_{s}$ is a homeomorphism from $D_{s^{*} s}^{\alpha}$ to $D_{s s^{*}}^{\alpha}$. In this paper, we always assume that $\bigcup_{e \in E(S)} D_{e}^{\alpha}=X$ holds.

For an action $\alpha: S \curvearrowright X$, we associate an étale groupoid $S \ltimes_{\beta} X$ as follows. First, we put the set $S * X:=\left\{(s, x) \in S \times X \mid x \in D_{s^{*} s}^{\alpha}\right\}$. Then we define an equivalence relation $\sim$ on $S * X$ by $(s, x) \sim(t, y)$ if

$$
x=y \text { and there exists } e \in E(S) \text { such that } x \in D_{e}^{\alpha} \text { and } s e=t e .
$$

Set $S \ltimes_{\alpha} X:=S * X / \sim$ and denote the equivalence class of $(s, x) \in S * X$ by $[s, x]$. The unit space $S \ltimes_{\alpha} X$ is $X$, where $X$ is identified with a subset of $S \ltimes_{\alpha} X$ via the injection

$$
X \ni x \mapsto[e, x] \in S \ltimes_{\alpha} X, x \in D_{e}^{\alpha} .
$$

The source and range maps are defined by

$$
d([s, x])=x, r([s, x])=\alpha_{s}(x)
$$


for $[s, x] \in S \ltimes_{\alpha} X$. The product of $\left[s, \alpha_{t}(x)\right],[t, x] \in S \ltimes_{\alpha} X$ is [st, $\left.x\right]$. The inverse is $[s, x]^{-1}=\left[s^{*}, \alpha_{s}(x)\right]$. Then $S \ltimes_{\alpha} X$ is a groupoid with these operations. For $s \in S$ and an open set $U \subset D_{s^{*} s}^{\alpha}$, define

$$
[s, U]:=\left\{[s, x] \in S \ltimes_{\alpha} X \mid x \in U\right\} .
$$

These sets form an open basis of $S \ltimes_{\alpha} X$. With this structure, $S \ltimes_{\alpha} X$ is an étale groupoid.

Let $S$ be an inverse semigroup. Now we define the spectral action $\beta: S \curvearrowright \widehat{E(S)}$. A character on $E(S)$ is a nonzero semigroup homomorphism from $E(S)$ to $\{0,1\}$, where $\{0,1\}$ is an inverse semigroup with the usual product. The set of all characters on $E(S)$ is denoted by $\widehat{E(S)}$. We view $\widehat{E(S)}$ as a locally compact Hausdorff space with respect to the topology of pointwise convergence. Define

$$
N_{P}^{e}:=\{\xi \in \widehat{E(S)} \mid \xi(e)=1, \xi(p)=0 \text { for all } p \in P\}
$$

for $e \in E(S)$ and a finite subset $P \subset E(S)$. Then these sets form a basis for the topology on $\widehat{E(S)}$. For $e \in E(S)$, we define $D_{e}^{\beta}:=\{\xi \in \widehat{E(S)} \mid \xi(e)=1\}$. For each $s \in S$ and $\xi \in$ $D_{s^{*} s}^{\beta}$, define $\beta_{s}(\xi) \in D_{s s^{*}}^{\beta}$ by $\beta_{s}(\xi)(e)=\xi\left(s^{*} e s\right)$, where $e \in E(S)$. Then $\beta$ is an action $\beta: S \curvearrowright \widehat{E(S)}$, which we call the spectral action of $S$. Now the universal groupoid of $S$ is defined to be $G_{u}(S):=S \ltimes_{\beta} \widehat{E(S)}$.

\section{Certain least congruences}

It is known that every inverse semigroup admits the least Clifford congruence and the least commutative congruence. For example, see [5, Ch. III, Proposition 6.7] for the least Clifford congruence and [6] for the least commutative congruence. In this section, we reprove that every inverse semigroup admits the least Clifford congruence and the least commutative congruence by a new method using the spectrum.

3.1. Invariant subset of $\widehat{\boldsymbol{E}(\boldsymbol{S})}$. Let $S$ be an inverse semigroup. A subset $F \subset \widehat{E(S)}$ is said to be invariant if $\beta_{s}\left(F \cap D_{s^{*} s}\right) \subset F$ holds for all $s \in S$. Note that $F$ is invariant if and only if $F$ is invariant as a subset of the groupoid $G_{u}(S)$. We omit the proof of the next proposition.

PROPOSITION 3.1. Let $S$ be an inverse semigroup and $F \subset \widehat{E(S)}$ be an invariant subset. We define the set $\rho_{F} \subset E(S) \times E(S)$ of all pairs $(e, f) \in E(S) \times E(S)$ such that $\xi(e)=$ $\xi(f)$ holds for all $\xi \in F$. Then $\rho_{F}$ is a normal congruence on $E(S)$.

Let $S$ be an inverse semigroup and $\rho$ be a normal congruence on $E(S)$. Moreover, let $q: E(S) \rightarrow E(S) / \rho$ denote the quotient map. For $\xi \in E(S) / \rho$, we define $\widehat{q}(\xi) \in \widehat{E(S)}$ by $\widehat{q}(\xi)(e)=\xi(q(e))$, where $e \in E(S)$. Note that $\widehat{q}(\xi)$ is not zero since $q$ is surjective. Then $\widehat{q}: \widehat{E(S) / \rho} \rightarrow \widehat{E(S)}$ is a continuous map by the definition of the topology of pointwise 
convergence. One can see that

$$
\widehat{q}(\widehat{E(S) / \rho})=\bigcap_{(e, f) \in \rho}\{\xi \in \widehat{E(S)} \mid \xi(e)=\xi(f)\}
$$

holds. In particular, $F_{\rho}:=\widehat{q}(\widehat{E(S) / \rho})$ is a closed subset of $\widehat{E(S)}$.

We say that $F \subset \widehat{E(S)}$ is multiplicative if the multiplication of two elements in $F$ also belongs to $F$ whenever it is not zero.

PROPOSITION 3.2. Let $S$ be an inverse semigroup and $\rho$ be a normal congruence on $E(S)$. Then $F_{\rho} \subset \widehat{E(S)}$ is a closed multiplicative invariant set.

Proof. It is easy to show that $F_{\rho} \subset \widehat{E(S)}$ is a closed multiplicative set. We show that $F_{\rho} \subset \widehat{E(S)}$ is invariant. Take $\xi \in F_{\rho}$ and $s \in S$ with $\xi\left(s^{*} s\right)=1$. By the definition of $F_{\rho}$, there exists $\eta \in \widehat{E(S) / \rho}$ such that $\xi=\eta \circ q$, where $q: E(S) \rightarrow E(S) / \rho$ denotes the quotient map. Then

$$
\beta_{s}(\xi)(e)=\xi\left(s^{*} e s\right)=\eta\left(q\left(s^{*} e s\right)\right)=\beta_{q(s)}(\eta)(q(e))=\widehat{q}\left(\beta_{q(s)}(\eta)\right)(e)
$$

for all $e \in E(S)$. Therefore, it follows that $\beta_{s}(\xi)=\widehat{q}\left(\beta_{q(s)}(\eta)\right) \in F_{\rho}$.

Proposition 3.3. Let $S$ be an inverse semigroup. Then $\rho=\rho_{F_{\rho}}$ holds for every normal congruence $\rho$ on $E(S)$.

Proof. Assume that $(e, f) \in \rho$. For all $\eta \in \widehat{E(S) / \rho \text {, }}$

$$
\widehat{q}(\eta)(e)=\eta(q(e))=\eta(q(f))=\widehat{q}(\eta)(f) .
$$

Therefore, $(e, f) \in \rho_{F_{\rho}}$.

To show the reverse inclusion, assume that $(e, f) \in \rho_{F_{\rho}}$. Define $\eta_{q(e)} \in \widehat{E(S) / \rho}$ by

$$
\eta_{q(e)}(p)= \begin{cases}1 & (p \geq q(e)) \\ 0 & \text { (otherwise) }\end{cases}
$$

where $p \in E(S) / \rho$. By $(e, f) \in \rho_{F_{\rho}}$, we have $\eta_{q(e)}(q(f))=\eta_{q(e)}(q(e))=1$. Therefore, $q(f) \geq q(e)$. Similarly we obtain $q(f) \leq q(e)$, so $q(e)=q(f)$ holds. Thus, it follows that $(e, f) \in \rho$.

We say that $F \subset \widehat{E(S)}$ is unital if $F$ contains the constant function 1 .

LEMMA 3.4. Let $S$ be an inverse semigroup and $F \subset \widehat{E(S)}$ be a unital multiplicative set. Assume that $F$ separates $E(S)$ (that is, for $e, f \in E(S), e=f$ is equivalent to the condition that $\xi(e)=\xi(f)$ holds for all $\xi \in F)$. Then $F$ is dense in $\widehat{E(S)}$.

Proof. For $e \in E(S)$ and a finite subset $P \subset E(S)$, we define

$$
N_{P}^{e}:=\{\xi \in \widehat{E(S)} \mid \xi(e)=1, \xi(p)=0 \text { for all } p \in P\} .
$$

Recall that these sets form an open basis of $\widehat{E(S)}$. Observe that $N_{P}^{e}=N_{e P}^{e}$ holds, where $e P:=\{e p \in E(S) \mid p \in P\}$. Now it suffices to show that $F \cap N_{P}^{e} \neq \emptyset$ holds for nonempty $N_{P}^{e}$ such that $p \leq e$ holds for all $p \in P$. 
In the case that $P=\emptyset$, the constant function 1 belongs to $F \cap N_{P}^{e}$. We may assume that $p \leq e$ holds for all $p \in P$. Since $N_{P}^{e}$ is nonempty, we have $e \neq p$ for all $p \in P$. Since $F$ separates $E(S)$, there exists $\xi_{p} \in F$ such that $\xi_{p}(e)=1$ and $\xi_{p}(p)=0$ for each $p \in P$. Define $\xi:=\prod_{p \in P} \xi_{p}$; then $\xi \in N_{P}^{e} \cap F$.

PROPOSITION 3.5. Let $S$ be an inverse semigroup. Then $F=F_{\rho_{F}}$ holds for every unital multiplicative invariant closed set $F \subset \widehat{E(S)}$.

PROOF. It is easy to show that $F \subset F_{\rho_{F}}$. Let $q: E(S) \rightarrow E(S) / \rho_{F}$ denote the quotient map. Then the set $\widehat{q}^{-1}(F)$ is a unital multiplicative closed set which separates $E(S) / \rho_{F}$. By Lemma 3.4, $\widehat{q}^{-1}(F)=E \widehat{(S) / \rho_{F}}$ holds. Therefore, we have $F \supset \widehat{q}\left(\widehat{q}^{-1}(F)\right)=$ $\widehat{q}\left(E \overline{(S) / \rho_{F}}\right)=F_{\rho_{F}}$.

COROLlARY 3.6. Let $S$ be an inverse semigroup. There is a one-to-one correspondence between normal congruences on $E(S)$ and unital multiplicative invariant closed sets in $\widehat{E(S)}$.

PROOF. Just combine Propositions 3.3 and 3.5.

3.2. The least Clifford congruences. Let $S$ be an inverse semigroup. Recall that a congruence $\rho$ on $S$ is said to be Clifford if $S / \rho$ is Clifford. For example, $S \times S$ is a Clifford congruence on $S$. In this subsection, we prove that every inverse semigroup admits the least Clifford congruence (Theorem 3.11). Our construction of the congruence is based on the fixed points of $\widehat{E(S)}$.

DEFinition 3.7. Let $S$ be an inverse semigroup. A character $\xi \in \widehat{E(S)}$ is said to be fixed if $\xi\left(s^{*} e s\right)=\xi(e)$ holds for all $e \in E(S)$ and $s \in S$ such that $\xi\left(s^{*} s\right)=1$. We denote the set of all fixed characters by $\widehat{E(S)_{\text {fix }}}$.

One can see that $\widehat{E(S)}_{\text {fix }}$ is a closed subset of $\widehat{E(S)}$. Moreover, $\widehat{E(S)_{\text {fix }}}$ is a multiplicative set. The fixed characters are characterized in the next proposition.

Proposition 3.8. Let $S$ be an inverse semigroup. Then $\xi \in \widehat{E(S)}$ is fixed if and only if $\xi$ can be extended to a semigroup homomorphism $\widetilde{\xi}: S \rightarrow\{0,1\}$. In this case, $\widetilde{\xi}: S \rightarrow$ $\{0,1\}$ is the unique extension of $\xi$.

PRoOF. If $\xi \in \widehat{E(S)}$ has an extension $\widetilde{\xi}: S \rightarrow\{0,1\}$,

$$
\widetilde{\xi}(s)=\widetilde{\xi}(s)^{2}=\widetilde{\xi}\left(s^{*} s\right)=\xi\left(s^{*} s\right)
$$

for all $s \in S$. Therefore, a semigroup homomorphism extension of $\xi$ is unique if it exists.

It is obvious that $\xi$ is fixed if $\xi$ has a semigroup homomorphism extension. Assume that $\xi \in \widehat{E(S)}$ is fixed. Then define $\widetilde{\xi}(s): S \rightarrow\{0,1\}$ by $\widetilde{\xi}(s):=\xi\left(s^{*} s\right)$ for $s \in S$. For $s, t \in S$, if $\xi\left(t^{*} t\right)=1$, we have $\widetilde{\xi}(s t)=\xi\left(t^{*} s^{*} s t\right)=\xi\left(s^{*} s\right)=\widetilde{\xi}(s) \widetilde{\xi}(t)$. If $\xi\left(t^{*} t\right)=0$, we have $\widetilde{\xi}(s t)=\widetilde{\xi}(s) \widetilde{\xi}(t)=0$. Thus, $\widetilde{\xi}$ is a semigroup homomorphism. 
DEFINITION 3.9. Let $S$ be an inverse semigroup. We define the normal congruence $\rho_{\text {Clif }}:=\rho_{\widetilde{E(S)}_{\text {fix }}}$ on $E(S)$. Furthermore, we define the congruence $v_{\mathrm{Clif}}:=v_{\rho_{\mathrm{Clif}}, \text { min }}$ on $S$ and $S^{\text {Clif }}:=S / v_{\text {Clif }}$.

LEMMA 3.10. Let $S$ be an inverse semigroup, $v$ be a Clifford congruence on $S$ and $q: S \rightarrow S / v$ be the quotient map. Then a set

$$
F_{v}=\{\xi \circ q \in \widehat{E(S)} \mid \xi \in \widehat{E(S / v)}\}
$$

is contained in $\widehat{E(S)})_{\mathrm{fix}}$. Moreover, $\left.\widehat{E(S)}\right)_{\mathrm{fix}}=\widehat{E(S)}$ holds if and only if $S$ is Clifford.

Proof. Take $\xi \in \widehat{E(S / v)}$ and assume that $\xi\left(q\left(s^{*} s\right)\right)=1$ for some $s \in S$. For all $e \in$ $E(S)$

$$
q\left(s^{*} e s\right)=q(e s)^{*} q(e s)=q(e s) q(e s)^{*}=q\left(e s s^{*} e\right)=q\left(s s^{*}\right) q(e)=q\left(s^{*} s\right) q(e)
$$

since $S / v$ is Clifford. Now

$$
\xi \circ q\left(s^{*} e s\right)=\xi\left(q\left(s^{*} e s\right)\right)=\xi\left(q\left(s^{*} s\right) q(e)\right)=\xi\left(q\left(s^{*} s\right)\right) \xi(q(e))=\xi \circ q(e) .
$$

Therefore, $\xi \circ q$ is a fixed character.

Applying what we have shown for the trivial congruence $v=\{(s, s) \in S \times S \mid s \in S\}$, it follows that $\widehat{E(S)_{\text {fix }}}=\widehat{E(S)}$ holds if $S$ is Clifford. Assume that $\widehat{E(S)_{\text {fix }}}=\widehat{E(S)}$ holds and take $s \in S$. Define a character $\xi_{s^{*} s} \in \widehat{E(S)}$ by

$$
\xi_{s^{*} s}(e)= \begin{cases}1 & \left(e \geq s^{*} s\right) \\ 0 & (\text { otherwise })\end{cases}
$$

where $e \in E(S)$. Since we assume that $\widehat{E(S)_{\text {fix }}}=\widehat{E(S)}$ and $\xi_{s^{*} s}\left(s^{*} s\right)=1$,

$$
\xi_{s^{*} s}\left(s s^{*}\right)=\xi_{s^{*} s}\left(s^{*}\left(s s^{*}\right) s\right)=\xi_{s^{*} s}\left(s^{*} s\right)=1 .
$$

Then we have $s^{*} s \leq s s^{*}$. It follows that $s^{*} s \geq s s^{*}$ from the same argument. Now we have $s^{*} s=s s^{*}$ and $S$ is Clifford.

Now we show that every inverse semigroup admits the Cliffordization.

THEOREM 3.11. Let $S$ be an inverse semigroup. Then $v_{\text {Clif }}$ is the least Clifford congruence on $S$.

Proof. First, we show that the congruence $v_{\text {Clif }}$ is Clifford. Take $s \in S$ and $\xi \in \widehat{E(S)_{\text {fix }}}$. Then one can see that $\xi\left(s^{*} s\right)=\xi\left(s s^{*}\right)$. Therefore, $\left(s^{*} s, s s^{*}\right) \in v_{\mathrm{Clif}}$ and $v_{\mathrm{Clif}}$ is a Clifford congruence.

Let $v$ be a Clifford congruence and $q: S \rightarrow S / v$ be the quotient map. To show that $v_{\text {Clif }} \subset v$, take $(s, t) \in v_{\text {Clif }}$. First, we show that $\left(s^{*} s, t^{*} t\right) \in v$. We define $\eta \in \widehat{E(S / v)}$ by

$$
\eta(e)= \begin{cases}1 & \left(e \geq q\left(s^{*} s\right)\right) \\ 0 & (\text { otherwise })\end{cases}
$$


By Lemma 3.10, it follows that $\eta \circ q \in \widehat{E(S}_{\text {fix }}$. Since $(s, t) \in v_{\text {Clif }}$, we have $1=\eta \circ$ $q\left(s^{*} s\right)=\eta \circ q\left(t^{*} t\right)$, which implies $q\left(t^{*} t\right) \geq q\left(s^{*} s\right)$. The reverse inequality is obtained symmetrically and therefore $q\left(t^{*} t\right)=q\left(s^{*} s\right)$ holds.

Let $\eta \in E\left(\widehat{S / v_{\text {Clif }}}\right)$ be the above character. Since $\eta \circ q$ is a fixed character and $(s, t) \in$ $v_{\text {Clif }}$, there exists $e \in E(S)$ such that $\eta \circ q(e)=1$ and $s e=t e$ hold. Since $\eta \circ q(e)=1$, we have $q(e) \geq q\left(s^{*} s\right)=q\left(t^{*} t\right)$ by the definition of $\eta$. Now we have $q(s)=q(s) q(e)=$ $q(t) q(e)=q(t)$. Therefore, $(s, t) \in v$.

COROLlaRY 3.12. Let $S$ be an inverse semigroup, $T$ be a Clifford inverse semigroup and $\varphi: S \rightarrow T$ be a semigroup homomorphism. Then there exists a unique semigroup homomorphism $\widetilde{\varphi}: S^{\text {Clif }} \rightarrow$ T that makes the following diagram commutative:

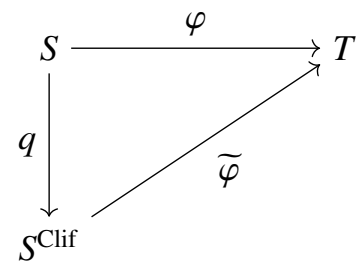

where $q: S \rightarrow S^{\text {Clif }}$ denotes the quotient map.

3.3. The least commutative congruences. We say that a congruence on an inverse semigroup is commutative if the quotient semigroup is commutative. In this subsection, we show that every inverse semigroup admits the least commutative congruence.

We denote the circle group by $\mathbb{T}:=\{z \in \mathbb{C}|| z \mid=1\}$. We view $\mathbb{T} \cup\{0\}$ as an inverse semigroup with the usual products. By $\widehat{S}$, we denote the set of all semigroup homomorphisms from $S$ to $\mathbb{T} \cup\{0\}$.

Definition 3.13. Let $S$ be an inverse semigroup. We define the commutative congruence $v_{\mathrm{ab}}$ on $S$ as the set of all pairs $(s, t) \in S \times S$ such that $\varphi(s)=\varphi(t)$ holds for all $\varphi \in \widehat{S}$. We define $S^{\mathrm{ab}}:=S / \nu_{\mathrm{ab}}$.

Let $S$ be a Clifford inverse semigroup and $e \in E(S)$. We define $H_{e}:=\left\{s \in S \mid s^{*} s=e\right\}$. One can see that $H_{e}$ is a group with the operation inherited from $S$. Note that the unit of $H_{e}$ is $e$.

In order to show that $v_{\mathrm{ab}}$ is the least commutative congruence, we need the next lemma.

LEMMA 3.14. Let $S$ be a Clifford inverse semigroup and $e \in E(S)$. Then a group homomorphism $\varphi: H_{e} \rightarrow \mathbb{T}$ can be extended to a semigroup homomorphism $\widetilde{\varphi}: S \rightarrow$ $\mathbb{T} \cup\{0\}$.

Proof. Define

$$
\widetilde{\varphi}(s)= \begin{cases}\varphi(s e) & \left(s^{*} s \geq e\right) \\ 0 & (\text { otherwise })\end{cases}
$$

Then one can check that $\tilde{\varphi}$ is a semigroup homomorphism extension of $\varphi$. 
THEOREM 3.15. Let $S$ be an inverse semigroup. Then $v_{\mathrm{ab}}$ is the least commutative congruence on $S$.

Proof. Assume that $v$ is a commutative congruence. Let $q: S \rightarrow S / v$ denote the quotient map. In order to show that $v_{\mathrm{ab}} \subset v$, take $(s, t) \in v_{\mathrm{ab}}$.

First, we show that $q\left(s^{*} s\right)=q\left(t^{*} t\right)$. It suffices to show that $\xi\left(q\left(s^{*} s\right)\right)=\xi\left(q\left(t^{*} t\right)\right)$ holds for all $\xi \in \widehat{E(S / v)}$. Note that $\xi \circ q \in \widehat{E(S)}$ is a fixed point by Lemma 3.10. Since $\xi \circ q$ is a restriction of an element in $\widehat{S}$ by Proposition 3.8, $\xi\left(q\left(s^{*} s\right)\right)=\xi\left(q\left(t^{*} t\right)\right)$ follows from $\left(s^{*} s, t^{*} t\right) \in v_{\mathrm{ab}}$.

In order to show that $q(s)=q(t)$, it suffices to show that $\psi(q(s))=\psi(q(t))$ for all group homomorphisms $\psi: H_{q\left(s^{*} s\right)} \rightarrow \mathbb{T}$ since $H_{q\left(s^{*} s\right)}=\left\{a \in S / v \mid a^{*} a=q\left(s^{*} s\right)\right\}$ is an abelian group. By Lemma 3.14, there exists a semigroup homomorphism extension $\widetilde{\psi} \in \widehat{S / v}$ of $\psi$. Since $\widetilde{\psi} \circ q \in \widehat{S}$ and $(s, t) \in v_{\mathrm{ab}}$, we have $\psi(q(s))=\psi(q(t))$. Therefore, $q(s)=q(t)$ holds.

COROLlary 3.16. Let $S$ be an inverse semigroup, $T$ be a commutative inverse semigroup and $\varphi: S \rightarrow T$ be a semigroup homomorphism. Then there exists a unique semigroup homomorphism $\widetilde{\varphi}: S^{\mathrm{ab}} \rightarrow T$ that makes the following diagram commutative:

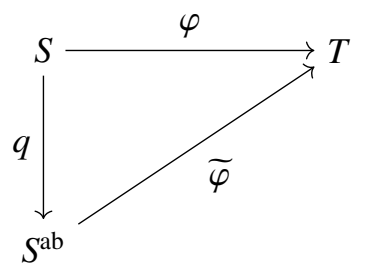

where $q: S \rightarrow S^{\mathrm{ab}}$ denotes the quotient map.

\section{Universal étale groupoids associated to quotient inverse semigroups}

4.1. General case. Let $S$ be an inverse semigroup and $v$ be a congruence on $S$. Let $q: S \rightarrow S / v$ denote the quotient map. Note that

$$
F_{v}=\{\xi \circ q \in E(S) \mid \xi \in \widehat{E(S)}\}
$$

is a closed invariant subset of $G_{u}(S)$ as shown in Proposition 3.2.

We omit the proof of the next proposition.

PROPOSITION 4.1. Let $S$ be an inverse semigroup and $H \subset S$ be a subsemigroup such that $E(S) \subset H$. Then the map

$$
G_{u}(H) \ni[s, \xi] \mapsto[s, \xi] \in G_{u}(S)
$$

is a groupoid homomorphism which is a homeomorphism onto its image. Moreover, the image is an open subgroupoid of $G_{u}(S)$. 
Via the map in the above proposition, we identify $G_{u}(H)$ with an open subgroupoid of $G_{u}(S)$.

Let $S$ be an inverse semigroup, $v$ be a congruence on $S$ and $q: S \rightarrow S / v$ be the quotient map. Define $\operatorname{ker} v:=q^{-1}(E(S / v)) \subset S$. Then $\operatorname{ker} v$ is a normal subsemigroup of $S$. Although $G_{u}(\operatorname{ker} v)$ is not necessarily a normal subgroupoid of $G_{u}(S)$, the following holds.

PROPOSITION 4.2. Let $S$ be an inverse semigroup and $v$ be a congruence on $S$. Then $G_{u}(\operatorname{ker} v)_{F_{v}}$ is an open normal subgroupoid of $G_{u}(S)_{F_{v}}$.

PROOF. We know that $G_{u}(\operatorname{ker} v)_{F_{v}}$ is an open normal subgroupoid of $G_{u}(S)_{F_{v}}$. We show that $G_{u}(\operatorname{ker} v)_{F_{v}}$ is normal in $G_{u}(S)_{F_{v}}$. Let $q: S \rightarrow S / v$ denote the quotient map.

First, we show that $G_{u}(\operatorname{ker} v)_{F_{v}} \subset \operatorname{Iso}\left(G_{u}(S)_{F_{v}}\right)$. Take $[n, \xi] \in G_{u}(\operatorname{ker} v)_{F_{v}}$, where $n \in \operatorname{ker} v$. Since $\xi \in F_{v}$ holds, there exists $\eta \in \widehat{E(S / v)}$ such that $\xi=\eta \circ q$. Since $q(n) \in$ $E(S / v)$ holds, we have $q\left(n^{*}\right) \in E(S / v)$ and

$$
\begin{aligned}
\beta_{n}(\xi)(e) & =\xi\left(n^{*} e n\right)=\eta\left(q\left(n^{*}\right) q(e) q(n)\right) \\
& =\eta\left(q\left(n^{*}\right)\right) \eta(q(e)) \eta(q(n))=\eta\left(q\left(n^{*} n\right)\right) \eta(q(e))=\xi(e)
\end{aligned}
$$

for all $e \in E(S)$. Therefore, $\beta_{n}(\xi)=\xi$ holds and it follows that $[n, \xi] \in \operatorname{Iso}\left(G_{u}(\operatorname{ker} v)_{F_{v}}\right)$.

Next we show that $[s, \eta][n, \xi][s, \eta]^{-1} \in G_{u}(\operatorname{ker} v)_{F_{v}}$ holds for all $[n, \xi] \in G_{u}(\operatorname{ker} v)_{F_{v}}$ and $[s, \eta] \in G_{u}(S)_{F_{v}}$ such that $\eta=\beta_{n}(\xi)$. One can see that

$$
[s, \eta][n, \xi][s, \eta]^{-1}=\left[s n s^{*}, \beta_{s}(\eta)\right] .
$$

Now it follows that $[s, \eta][n, \xi][s, \eta]^{-1} \in G_{u}(\operatorname{ker} v)_{F_{v}}$ from $s n s^{*} \in \operatorname{ker} v$.

THEOREM 4.3. Let $S$ be an inverse semigroup and $v$ be a congruence on $S$. Then $G_{u}(S / v)$ is isomorphic to $G_{u}(S)_{F_{v}} / G_{u}(\operatorname{ker} v)_{F_{v}}$.

ProOF. Let $q: S \rightarrow S / v$ denote the quotient map. Note that a map

$$
\widehat{q}: \widehat{E(S / v)} \ni \xi \mapsto \xi \circ q \in F_{v}
$$

is a homeomorphism. Define a map

$$
\Phi: G_{u}(S)_{F_{v}} \ni[s, \widehat{q}(\xi)] \mapsto[q(s), \xi] \in G_{u}(S / v) .
$$

Using Proposition 2.1, one can see that $\Phi$ is a groupoid homomorphism which is a local homeomorphism and injective on $G_{u}(S)_{F_{v}}^{(0)}$. Observe that $\Phi$ is surjective.

We show that $\operatorname{ker} \Phi=G_{u}(\operatorname{ker} v)_{F_{v}}$ holds. The inclusion $\operatorname{ker} \Phi \supset G_{u}(\operatorname{ker} v)_{F_{v}}$ is obvious. In order to show that $\operatorname{ker} \Phi \subset G_{u}(\operatorname{ker} v)_{F_{v}}$, take $[s, \widehat{q}(\xi)] \in \operatorname{ker} \Phi$. Since we have $[q(s), \xi] \in G_{u}(S / v)^{(0)}$ and $q(E(S))=E(S / v)$, there exists $e \in E(S)$ such that $[q(s), \xi]=$ $[q(e), \xi]$. There exists $f \in E(S)$ such that $\xi(q(f))=1$ and $q(s) q(f)=q(e) q(f)$. Now we have $s f \in \operatorname{ker} v$, so it follows that

$$
[s, \widehat{q}(\xi)]=[s f, \widehat{q}(\xi)] \in G_{u}(\operatorname{ker} v)_{F_{v}} .
$$

This shows that $\operatorname{ker} \Phi=G_{u}(\operatorname{ker} v)_{F_{v}}$. 
By Proposition 2.2, $\Phi$ induces an isomorphism $\widetilde{\Phi}$ that makes the following diagram commutative:

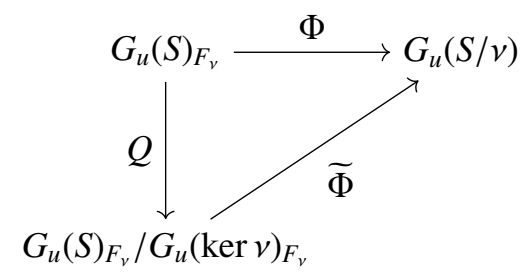

where $Q$ denotes the quotient map.

\subsection{Universal groupoids associated to special quotient inverse semigroups.}

4.2.1. Minimum congruences associated to normal congruences on semilattices of idempotents. Let $S$ be an inverse semigroup. Recall that a congruence $\rho$ on $E(S)$ is normal if $(e, f) \in \rho$ implies $\left(\operatorname{ses}^{*}, s f s^{*}\right) \in \rho$ for all $s \in S$ and $e, f \in E(S)$. Note that one can construct the least congruence $v_{\rho \text {, min }}$ whose restriction to $E(S)$ coincides with $\rho$. Recall that we can associate the closed invariant subset $F_{\rho}$ of $G_{u}(S)$ as shown in Proposition 3.2.

PROPOSITION 4.4. Let $S$ be an inverse semigroup and $\rho$ be a normal congruence on $E(S)$. Then $G_{u}\left(S / v_{\rho, \min }\right)$ is isomorphic to $G_{u}(S)_{F_{\rho}}$.

PROOF. By Theorem 4.3, it suffices to show that $G_{u}\left(\operatorname{ker} v_{\rho, \min }\right)_{F_{\rho}}=G_{u}(S)_{F_{\rho}}^{(0)}$ holds. Let $q: S \rightarrow S / v_{\rho \text {, min }}$ denote the quotient map. Take $[n, \widehat{q}(\xi)] \in G_{u}\left(\operatorname{ker} v_{\rho \text {, min }}\right)_{F_{\rho}}$, where $n \in \operatorname{ker} v_{\rho, \min }$ and $\xi \in \widehat{E(S / \rho)}$. Since $n \in \operatorname{ker} v_{\rho, \min }$, there exists $e \in E(S)$ such that $q(n)=$ $q(e)$. By the definition of $v_{\rho \text {,min }}$, there exists $f \in E(S)$ such that $n f=e f$ and $\left(n^{*} n, f\right) \in \rho$ hold. Observe that $\widehat{q}(\xi)\left(n^{*} n\right)=\xi\left(q\left(n^{*} n\right)\right)=\xi(q(f))=\xi(q(e))=1$. We have

$$
[n, \widehat{q}(\xi)]=[n f, \widehat{q}(\xi)]=[e f, \widehat{q}(\xi)] \in G_{u}(S)_{F_{\rho}}^{(0)} .
$$

Now we have shown that $G_{u}\left(\operatorname{ker} v_{\rho, \min }\right)_{F_{\rho}}=G_{u}\left(\operatorname{ker} v_{\rho, \min }\right)_{F_{\rho}}^{(0)}$.

THEOREM 4.5. Let $S$ be an inverse semigroup. Then $G_{u}\left(S^{\text {Clif }}\right)$ is isomorphic to $G_{u}(S)_{\text {fix }}$.

PROOF. Recall the definition of $v_{\text {Clif }}=v_{\rho_{\text {Clif }} \text { min }}$ (see Definition 3.9). Since we have Proposition 4.4, it suffices to show that $F_{\rho_{\text {Clif }}}=\widehat{E(S}_{\text {fix }}$. By Lemma 3.10, we have $F_{\rho_{\text {Clif }}} \subset \widehat{E(S)_{\text {fix }}}$. To show the reverse inclusion, take $\xi \in \widehat{E(S)}_{\text {fix }}$. By Proposition 3.8, there exists a semigroup homomorphism extension $\widetilde{\xi}: S \rightarrow\{0,1\}$. Since $\{0,1\}$ is Clifford, there exists a semigroup homomorphism $\eta: S^{\text {Clif }} \rightarrow\{0,1\}$ such that $\eta \circ q=\widetilde{\xi}$, where $q: S \rightarrow S^{\text {Clif }}$ denotes the quotient map. Therefore, we have $\xi=\left.\eta \circ q\right|_{E(S)} \in F_{\rho_{\text {Clif }}}$. Now we have shown that $F_{\rho_{\text {Clif }}}=\widehat{E(S)}$ fix . 
4.2.2. The least commutative congruences. Let $S$ be an inverse semigroup and $v_{\mathrm{ab}}$ be the least commutative congruence (see Proposition 3.13 and Theorem 3.15). Recall that the abelianization of $S$ is defined to be $S^{\mathrm{ab}}:=S / \nu_{\mathrm{ab}}$.

THEOREM 4.6. Let $S$ be an inverse semigroup. Then $G_{u}\left(S^{\mathrm{ab}}\right)$ is isomorphic to $G_{u}(S)^{\mathrm{ab}}$.

PROOF. By Theorem 4.3, it suffices to show that $F_{v_{\mathrm{ab}}}=\widehat{E(S)}_{\mathrm{fix}}$ and $G_{u}\left(\operatorname{ker} v_{\mathrm{ab}}\right)_{\mathrm{fix}}=$ $\left[G_{u}(S)_{\text {fix }}, G_{u}(S)_{\text {fix }}\right]$ hold.

Observe that $v_{\mathrm{ab}}$ is equal to $v_{\mathrm{Clif}}$ on $E(S)$. Indeed, this follows from the fact that $\left.\varphi\right|_{E(S)} \in \widehat{E(S)}_{\text {fix }}$ holds for all $\varphi \in \widehat{S}$. Therefore, we have $\left.F_{v_{\mathrm{ab}}}=\widehat{E(S)}\right)_{\mathrm{fix}}$.

Next we show that $G_{u}\left(\operatorname{ker} v_{\mathrm{ab}}\right)_{\mathrm{fix}}=\left[G_{u}(S)_{\mathrm{fix}}, G_{u}(S)_{\mathrm{fix}}\right]$. The inclusion

$$
G_{u}\left(\operatorname{ker} v_{\mathrm{ab}}\right)_{\mathrm{fix}} \supset\left[G_{u}(S)_{\mathrm{fix}}, G_{u}(S)_{\mathrm{fix}}\right]
$$

is easy to show.

Let $q: S \rightarrow S^{\text {ab }}$ and $q^{\prime}: S \rightarrow S^{\text {Clif }}$ denote the quotient maps. Since a commutative inverse semigroup is Clifford, there exists a semigroup homomorphism $\sigma: S^{\mathrm{Clif}} \rightarrow S^{\mathrm{ab}}$ such that $q=\sigma \circ q^{\prime}$. To show the reverse inclusion

$$
G_{u}\left(\operatorname{ker} v_{\mathrm{ab}}\right)_{\mathrm{fix}} \subset\left[G_{u}(S)_{\mathrm{fix}}, G_{u}(S)_{\mathrm{fix}}\right],
$$

take $[n, \widehat{q}(\xi)] \in G_{u}\left(\operatorname{ker} v_{\mathrm{ab}}\right)_{\text {fix }}$, where $n \in \operatorname{ker} v_{\mathrm{ab}}$ and $\left.\xi \in \widehat{E\left(S^{\mathrm{ab}}\right)}\right)$. Since $n \in \operatorname{ker} v_{\mathrm{ab}}$, there exists $e \in E(S)$ such that $q(n)=q(e)$. Then we have $q\left(n^{*} n\right)=q(e)$. Since $v_{\mathrm{ab}}$ coincides with $v_{\text {Clif }}$ on $E(S)$, it follows that $q^{\prime}\left(n^{*} n\right)=q^{\prime}(e)$. Define

$$
H_{q^{\prime}(e)}=\left\{s \in S^{\text {Clif }} \mid s^{*} s=q^{\prime}(e)\right\} ;
$$

then $H_{q^{\prime}(e)}$ is a group in the operation inherited from $S^{\text {Clif }}$. Observe that a unit of $H_{q^{\prime}(e)}$ is $q^{\prime}(e)$ and we have $q^{\prime}(n) \in H_{q^{\prime}(e)}$. Fix a group homomorphism $\chi: H_{q^{\prime}(e)} \rightarrow \mathbb{T}$ arbitrarily. By Proposition 3.14, $\chi$ is extended to the semigroup homomorphism $\tilde{\chi}: S^{\text {Clif }} \rightarrow \mathbb{T} \cup$ $\{0\}$. Since $\mathbb{T} \cup\{0\}$ is commutative, there exists a semigroup homomorphism $\bar{\chi}: S^{\mathrm{ab}} \rightarrow$ $\mathbb{T} \cup\{0\}$ that makes the following diagram commutative:

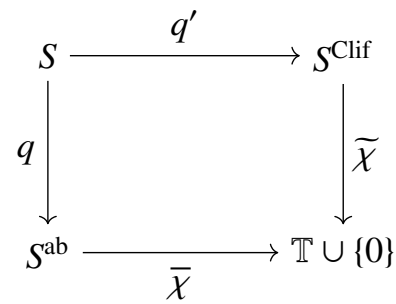

Now

$$
\chi\left(q^{\prime}(n)\right)=\bar{\chi}(q(n))=\bar{\chi}(q(e))=\chi\left(q^{\prime}(e)\right) .
$$

Since we take a group homomorphism $\chi: H_{q^{\prime}(e)} \rightarrow \mathbb{T}$ arbitrarily, it follows that $q^{\prime}(n) \in\left[H_{q^{\prime}(e)}, H_{q^{\prime}(e)}\right]$, where $\left[H_{q^{\prime}(e)}, H_{q^{\prime}(e)}\right]$ denotes the commutator subgroup of $H_{q^{\prime}(e)}$. 
Therefore, there exist $s_{1}, s_{2}, \ldots, s_{m}, t_{1}, t_{2}, \ldots, t_{m} \in S$ such that

$$
\begin{aligned}
q^{\prime}(n) & =q^{\prime}\left(s_{1}\right) q^{\prime}\left(t_{1}\right) q^{\prime}\left(s_{1}\right)^{*} q^{\prime}\left(t_{1}\right)^{*} \cdots q^{\prime}\left(s_{m}\right) q^{\prime}\left(t_{m}\right)^{\prime} q^{\prime}\left(s_{m}\right)^{*} q^{\prime}\left(t_{m}\right)^{*} \\
& =q^{\prime}\left(s_{1} t_{1} s_{1}^{*} t_{1}^{*} \cdots s_{m} t_{m} s_{m}^{*} t_{m}^{*}\right) .
\end{aligned}
$$

By the definition of $v_{\text {Clif }}$, there exists $f \in E(S)$ such that

$$
n f=s_{1} t_{1} s_{1}^{*} t_{1}^{*} \cdots s_{m} t_{m} s_{m}^{*} t_{m}^{*} f
$$

and $q^{\prime}\left(n^{*} n\right)=q^{\prime}(f)$ hold. Then

$$
\begin{aligned}
{[n, \widehat{q}(\xi)] } & =[n f, \widehat{q}(\xi)] \\
& =\left[s_{1} t_{1} s_{1}^{*} t_{1}^{*} \cdots s_{m} t_{m} s_{m}^{*} t_{m}^{*} f, \widehat{q}(\xi)\right] \\
& =\left[s_{1} t_{1} s_{1}^{*} t_{1}^{*} \cdots s_{m} t_{m} s_{m}^{*} t_{m}^{*}, \widehat{q}(\xi)\right] \in\left[G_{u}(S)_{\mathrm{fix}}, G_{u}(S)_{\mathrm{fix}}\right] .
\end{aligned}
$$

Thus, it is shown that $G_{u}\left(\operatorname{ker} v_{\mathrm{ab}}\right)_{\mathrm{fix}}=\left[G_{u}(S)_{\mathrm{fix}}, G_{u}(S)_{\mathrm{fix}}\right]$.

\section{Applications and examples}

5.1. Clifford inverse semigroups from the viewpoint of fixed points. A 0-group is an inverse semigroup isomorphic to $\Gamma \cup\{0\}$ for some group $\Gamma$. For a group $\Gamma$, we denote the 0 -group associated to $\Gamma$ by $\Gamma^{0}:=\Gamma \amalg\{0\}$. It is clear that every 0 -group is a Clifford inverse semigroup. Conversely, we see that every Clifford inverse semigroup is embedded into a direct product of 0-groups. We remark that this fact is already known (see [5, Theorem 2.6]). Using fixed characters, we obtain a new proof.

Let $S$ be a Clifford inverse semigroup and $\xi \in \widehat{E(S)}$. Since $\{\xi\} \subset \widehat{E(S)}$ is invariant by Lemma 3.10, we may consider a normal congruence $\rho_{\xi}:=\rho_{\{\xi\}}$ on $E(S)$ and a congruence $v_{\xi}:=v_{\rho_{\langle\xi\}}, \min }$ on $S$. If $\xi=1, \rho_{\xi}$ coincides with $E(S) \times E(S)$ and $S / v_{\xi}$ is the maximal group image of $S$. We define $S(\xi):=\left\{q_{\xi}(s) \in S / v_{\xi} \mid \xi\left(s^{*} s\right)=1\right\}$, where $q_{\xi}: S \rightarrow S / v_{\xi}$ is the quotient map. Then $S(\xi)$ is a group.

Define the map $\varphi_{\xi}: S \rightarrow S(\xi)^{0}$ by

$$
\varphi_{\xi}(s):= \begin{cases}q_{\xi}(s) & \left(\xi\left(s^{*} s\right)=1\right) \\ 0 & \left(\xi\left(s^{*} s\right)=0\right)\end{cases}
$$

Then $\varphi_{\xi}$ is a semigroup homomorphism.

Proposition 5.1. Let $S$ be a Clifford inverse semigroup. Then the semigroup homomorphism

$$
\Phi: S \ni s \mapsto\left(\varphi_{\xi}(s)\right)_{\xi \in \widehat{E(S)}} \in \prod_{\xi \in \widehat{E(S)}} S(\xi)^{0}
$$

is injective. In particular, every Clifford inverse semigroup is embedded into a direct product of 0-groups.

Proof. Assume that $s, t \in S$ satisfy $\Phi(s)=\Phi(t)$. Since we have $\varphi_{\xi}\left(s^{*} s\right)=\varphi_{\xi}\left(t^{*} t\right)$ for all $\xi \in \widehat{E(S)}$, it follows that $\xi\left(s^{*} s\right)=\xi\left(t^{*} t\right)$ for all $\xi \in \widehat{E(S)}$. Therefore, we obtain $s^{*} s=t^{*} t$. 
Define $\xi_{s^{*} s} \in \widehat{E(S)}$ by

$$
\xi_{s^{*} s}(e):= \begin{cases}1 & \left(e \geq s^{*} s\right), \\ 0 & (\text { otherwise }) .\end{cases}
$$

Then we have $\xi_{s^{*} s}\left(s^{*} s\right)=\xi_{s^{*} s}\left(t^{*} t\right)=1$. Combining with $\varphi_{\xi_{s^{*} s}}(s)=\varphi_{\xi_{s^{*} s}}(t)$, we obtain $q_{\xi_{s^{*} s}}(s)=q_{\xi_{s^{*} s}}(t)$. Therefore, there exists $e \in E(S)$ such that $\xi_{s^{*} s}(e)=\xi_{s^{*} s}\left(s^{*} s\right)=1$ and $s e=t e$. It follows that $e \geq s^{*} s\left(=t^{*} t\right)$ from $\xi_{s^{*} s}(e)=1$. Thus, we have shown that $s=t$ and $\Phi$ is injective.

Proposition 5.2. Let $S$ be a finitely generated Clifford inverse semigroup. Then $\widehat{E(S)}$ is a finite set.

Proof. Take a finite set $F \subset S$ which generates $S$. Let $X$ denote the set of all nonzero semigroup homomorphisms from $S$ to $\{0,1\}$. Then a map

$$
X \ni \xi \mapsto(\xi(f))_{f \in F} \in\{0,1\}^{F}
$$

is injective since $F$ generates $S$. By Proposition 3.8 and Lemma 3.10, the map $X \ni \xi \mapsto$ $\left.\xi\right|_{E(S)} \in \widehat{E(S)}$ is bijective. Since $\widehat{E(S)}$ is embedded into $\{0,1\}^{F}, \widehat{E(S)}$ is a finite set.

COROLlARY 5.3. Let $S$ be a finitely generated Clifford inverse semigroup. Then $S$ is embedded into a direct sum of finitely many 0-groups.

Let $S$ be a Clifford inverse semigroup and $\xi \in \widehat{E(S)}$. Recall that $G_{u}(S)_{\xi}$ is a discrete group. In [2], the authors gave a way to calculate $G_{u}(S)_{\xi}$. Then $\xi^{-1}(\{1\})$ is a directed set with respect to the order inherited from $E(S)$. For $e \in E(S)$, define $S(e):=\left\{s \in S \mid s^{*} s=e\right\}$. Then $S(e)$ is a group. For $e, f \in E(S)$ with $e \leq f$, define a map $\varphi_{e}^{f}: S(f) \rightarrow S(e)$ by $\varphi_{e}^{f}(s)=s e$ for $s \in S(f)$. Then $\varphi_{e}^{f}$ is a group homomorphism. One can see that $\left(S(e), \varphi_{e}^{f}\right)$ consists of an inductive system of groups. The authors proved the following theorem.

THEOREM 5.4 [2, Theorem 3.1]. Let $S$ be a Clifford inverse semigroup and $\xi \in \widehat{E(S)}$. Then there exists an isomorphism

$$
G_{u}(S)_{\xi} \simeq \underset{\xi(e)=1}{\lim _{\overrightarrow{1}}} S(e) .
$$

We give a way to realize $\lim _{\xi(e)=1} S(e)$ as a quotient of $S$.

PROPOSITION 5.5. Let $S$ be a Clifford inverse semigroup and $\xi \in \widehat{E(S)}$. Then we have the following isomorphism:

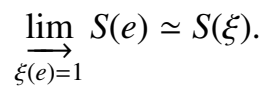

Proof. Let $\varphi_{\xi}: S \rightarrow S(\xi)^{0}$ denote the map in Proposition 5.1 and put $\Gamma:=\lim S(e)$. For $e \in E(S)$ with $\xi(e)=1$, we define $\sigma_{e}: S(e) \rightarrow S(\xi)$ by $\sigma_{e}(s):=\varphi_{\xi}(s)$. We $\overrightarrow{\text { obtain a }}$ group homomorphism $\widetilde{\sigma}: \Gamma \rightarrow S(\xi)$. One can see that $\widetilde{\sigma}$ is an isomorphism.

Combining Theorem 5.4 with Proposition 5.5, we obtain the next corollary. 
COROLlary 5.6. Let $S$ be a Clifford inverse semigroup and $\xi \in \widehat{E(S)}$. Then $G_{u}(S)_{\xi}$ is isomorphic to $S(\xi)$.

Let $I$ be a discrete set and $\left\{\Gamma_{i}\right\}_{i \in I}$ be a family of discrete groups. Then the disjoint union $\bigsqcup_{i \in I} \Gamma_{i}$ is a discrete group bundle over $I$ in the natural way. Using Proposition 5.2 and Corollary 5.6, we obtain the next corollary.

COROLlARY 5.7. Let $S$ be a finitely generated Clifford inverse semigroup. Then there exists an isomorphism

$$
G_{u}(S) \simeq \coprod_{\xi \in \widehat{E(S)}} S(\xi)
$$

For an étale groupoid $G$ with the locally compact Hausdorff unit space $G^{(0)}$, we write $C^{*}(G)$ (respectively $C_{\lambda}^{*}(G)$ ) to represent the universal (respectively reduced) groupoid $C^{*}$-algebra of $G$ (see [4] for the definitions). Corollary 5.7 immediately implies the next corollary.

COROLLARY 5.8. Let $S$ be a finitely generated Clifford inverse semigroup. Then we have isomorphisms

$$
C^{*}\left(G_{u}(S)\right) \simeq \bigoplus_{\xi \in \widehat{E(S)}} C^{*}(S(\xi)), \quad C_{\lambda}^{*}\left(G_{u}(S)\right) \simeq \bigoplus_{\xi \in \widehat{E(S)}} C_{\lambda}^{*}(S(\xi)) .
$$

5.2. Free Clifford inverse semigroups. We investigate universal groupoids and $\mathrm{C}^{*}$-algebras associated to free Clifford inverse semigroups on finite sets.

First, we recall the definition of the free groups.

Definition 5.9. Let $X$ be a set. A free group on $X$ is a pair $(\mathbb{F}(X), \kappa)$ consisting of a group $\mathbb{F}(X)$ and a map $\kappa: X \rightarrow \mathbb{F}(X)$ such that:

(1) $\quad \kappa(X)$ generates $\mathbb{F}(X)$; and

(2) for every group $\Gamma$ and a map $\varphi: X \rightarrow \Gamma$, there exists a group homomorphism $\widetilde{\varphi}: \mathbb{F}(X) \rightarrow \Gamma$ such that $\widetilde{\varphi}(x)=\varphi(\kappa(x))$ holds for all $x \in X$.

We define free inverse semigroups in a similar way.

Definition 5.10. Let $X$ be a set. A free inverse semigroup on $X$ is a pair $(\operatorname{FIS}(X), \iota)$ consisting of an inverse semigroup $\operatorname{FIS}(X)$ and a map $\iota: X \rightarrow \operatorname{FIS}(X)$ such that:

(1) $\iota(X)$ generates $\operatorname{FIS}(X)$; and

(2) for every inverse semigroup $T$ and a map $\varphi: X \rightarrow T$, there exists a semigroup homomorphism $\widetilde{\varphi}: \operatorname{FIS}(X) \rightarrow T$ such that $\widetilde{\varphi}(x)=\varphi(\iota(x))$ holds for all $x \in X$.

It is known that free inverse semigroups exist and are unique up to isomorphism. See [3, Section 6.1] for the existence of free inverse semigroups. The uniqueness is obvious. 
Definition 5.11. A free Clifford inverse semigroup on $X$ is a pair $(\operatorname{FCIS}(X), \iota)$ consisting of a Clifford inverse semigroup FCIS $(X)$ and a map $\iota: X \rightarrow \operatorname{FCIS}(X)$ such that:

(1) $\iota(X)$ generates FCIS $(X)$; and

(2) for every Clifford inverse semigroup $T$ and a map $\varphi: X \rightarrow T$, there exists a semigroup homomorphism $\widetilde{\varphi}: \operatorname{FCIS}(X) \rightarrow T$ such that $\widetilde{\varphi}(x)=\varphi(\iota(x))$ holds for all $x \in X$.

Free Clifford inverse semigroups exist and are unique up to isomorphism. Indeed, for a free Clifford inverse semigroup ( $\operatorname{FIS}(X), \iota)$ and the quotient map $q: \operatorname{FIS}(X) \rightarrow$ $\operatorname{FIS}(X)^{\text {Clif }}$, one can see that (FIS $\left.(X)^{\text {Clif }}, q \circ \iota\right)$ is a free Clifford inverse semigroup on $X$. The uniqueness is obvious.

Let $X$ be a set. For $A \subset X$, define a map $\chi_{A}: X \rightarrow\{0,1\}$ by

$$
\chi_{A}(x)= \begin{cases}1 & (x \in A), \\ 0 & (x \notin A) .\end{cases}
$$

Since $\{0,1\}$ is Clifford, $\chi_{A}$ can be extended to the semigroup homomorphism from $\operatorname{FCIS}(X)$ to $\{0,1\}$, which we also denote by $\chi_{A}$. Every semigroup homomorphism from FCIS $(X)$ to $\{0,1\}$ is of the form $\chi_{A}$ for a unique $A \subset X$.

By Proposition 3.8, $\left.\chi_{A}\right|_{E(\operatorname{FCIS}(X))}$ is a fixed character if $A$ is not empty. By Lemma 3.10 , all characters on $E(\mathrm{FCIS}(X))$ are fixed characters. Therefore, we obtain the next proposition.

Proposition 5.12. Let $X$ be a finite set. Put $S=\operatorname{FCIS}(X)$. Then the map

$$
\left.P(X) \backslash\{\emptyset\} \ni A \mapsto \chi_{A}\right|_{E(S)} \in \widehat{E(S)}
$$

is bijective, where $P(X)$ denotes the power set of $X$.

We identify $\left.\chi_{A}\right|_{E(\operatorname{FCIS}(X))}$ with $\chi_{A}$ since we can recover $\chi_{A}$ from the restriction $\left.\chi_{A} \mid \operatorname{E(FCIS}(X)\right)$.

For a nonempty set $A \subset X$, define $e_{A}:=\prod_{x \in A} \iota(x)^{*} \iota(x) \in E(\operatorname{FCIS}(X))$. For $e \in$ $E(\mathrm{FCIS}(X))$, the condition that $\chi_{A}(e)=1$ is equivalent to the condition that $e \geq e_{A}$. Using this fact, one can prove the next proposition.

Proposition 5.13. The map

$$
P(X) \backslash\{\emptyset\} \ni A \mapsto e_{A} \in E(\operatorname{FCIS}(X))
$$

is bijective.

In order to apply Corollary 5.6 for free Clifford inverse semigroups, we prepare with the next proposition.

Proposition 5.14. Let $X$ be $a$ set and $A \subset X$ be a nonempty set. Put $S=\operatorname{FCIS}(X)$. Then $S\left(\chi_{A}\right)$ is isomorphic to the free group $\mathbb{F}(A)$ generated by $A$. 
Proof. If $X=A, S\left(\chi_{A}\right)$ is the maximal group image of $S$. Therefore, $S\left(\chi_{A}\right)$ is isomorphic to $\mathbb{F}(A)$.

We assume that $A \subsetneq X$. Let $\varphi_{A}: S \rightarrow S\left(\chi_{A}\right)^{0}$ denote the map defined by

$$
\varphi_{A}(s)= \begin{cases}Q(s) & \left(\chi_{A}\left(s^{*} s\right)=1\right), \\ 0 & \left(\chi_{A}\left(s^{*} s\right)=0\right)\end{cases}
$$

where $Q: S \rightarrow S / \nu_{\chi_{A}}$ denotes the quotient map. By the universality of $\mathbb{F}(A)$, define a group homomorphism $\tau: \mathbb{F}(A) \rightarrow S\left(\chi_{A}\right)$ such that $\tau(\kappa(a))=\varphi_{A}(\iota(a))$ for all $a \in A$. We construct the inverse map of $\tau$. Using the universality of $S=\operatorname{FCIS}(X)$, define a semigroup homomorphism $\sigma: S \rightarrow \mathbb{F}(A)^{0}$ that satisfies

$$
\sigma(\iota(x))= \begin{cases}\kappa(x) & (x \in A), \\ 0 & (x \notin A)\end{cases}
$$

for $x \in X$. We claim that $(s, t) \in v_{\chi_{A}}$ implies $\sigma(s)=\sigma(t)$ for $s, t \in S$. If $\chi_{A}\left(s^{*} s\right)=0$, we have $\sigma(s)=\sigma(t)=0$. We may assume that $\chi_{A}\left(s^{*} s\right)=1$. By $(s, t) \in v_{\chi_{A}}$, we have $s e_{A}=t e_{A}$. Since $\sigma\left(e_{A}\right)$ is the unit of $\mathbb{F}(A)$, we have $\sigma(s)=\sigma(t)$. Therefore, we obtain a semigroup homomorphism $\widetilde{\sigma}: S\left(\chi_{A}\right)^{0} \rightarrow \mathbb{F}(A)^{0}$ that makes the following diagram commutative:

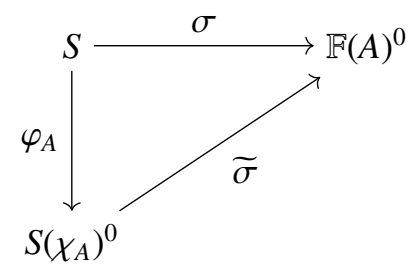

Now one can verify that $\left.\widetilde{\sigma}\right|_{S\left(\chi_{A}\right)}$ is the inverse map of $\tau$.

Now we have the following theorem.

THEOREM 5.15. Let $X$ be a finite set. Then there exists an isomorphism

$$
G_{u}(\operatorname{FCIS}(X)) \simeq \coprod_{A \in P(X) \backslash\{\emptyset\}} \mathbb{F}(A) .
$$

Proof. Put $S=$ FCIS $(X)$. By Proposition 5.12,

$$
\widehat{E(S)}=\left\{\chi_{A} \in \widehat{E(S)} \mid A \in P(X) \backslash\{\emptyset\}\right\}
$$

is a finite set. Therefore, we have an isomorphism

$$
G_{u}(S) \simeq \coprod_{A \in P(X) \backslash\{\emptyset\}} G_{u}(S)_{\chi_{A}} .
$$

By Proposition 5.14, we obtain the isomorphism in the statement. 
5.3. Fixed points of Boolean actions. From [9, Section 5], we recall the notion of Boolean actions. By a locally compact Boolean space, we mean a locally compact Hausdorff space that admits a basis of compact open sets. Let $S$ be an inverse semigroup and $X$ be a locally compact Boolean space. An action $\alpha: S \curvearrowright X$ is said to be Boolean if:

(1) for all $e \in E(S), D_{e}^{\alpha} \subset X$ is a compact open set of $X$; and

(2) the family

$$
\left\{D_{e}^{\alpha} \cup \bigcup_{f \in P}\left(X \backslash D_{f}^{\alpha}\right) \mid e \in E(S), P \subset E(S) \text { is a finite set }\right\}
$$

forms a basis of $X$.

It is known that $G_{u}(S)$ has the following universal property for Boolean actions.

TheOrem 5.16 [9, Proposition 5.5]. Let $S$ be an inverse semigroup, $X$ be a Boolean space and $\alpha: S \curvearrowright X$ be a Boolean action. Then $S \ltimes_{\alpha} X$ is isomorphic to $G_{u}(S)_{F}$ for some closed invariant subset $F \subset \widehat{E(S)}$.

COROLlary 5.17. Let $S$ be a finitely generated inverse semigroup and $\alpha: S \curvearrowright X$ be a Boolean action. Then $\alpha$ has finitely many fixed points. More precisely, the number of fixed points of $\alpha$ is less than or equal to the number of nonzero semigroup homomorphisms from $S$ to $\{0,1\}$.

PROOF. Since we assume that $S$ is finitely generated, the set of all nonzero semigroup homomorphisms from $S$ to $\{0,1\}$ is a finite set. By Proposition 3.8, there exists a bijection between the set of all nonzero semigroup homomorphisms from $S$ to $\{0,1\}$ and $\widehat{E(S)}_{\text {fix }}$. Now Theorem 5.16 completes the proof.

EXAMPLE 1 (cf. [4, Example 3 in Section 4.2]). For a natural number $n \in \mathbb{N}$, the Cuntz inverse semigroup $S_{n}$ is an inverse semigroup which is generated by $\left\{0,1, s_{1}, \ldots, s_{n}\right\}$ with the relation

$$
s_{i}^{*} s_{j}=\delta_{i, j} 1
$$

for all $i, j \in\{1,2, \ldots, n\}$. Define $\xi: S_{n} \rightarrow\{0,1\}$ by $\xi(x)=1$ for all $x \in S_{n}$. Then $\xi$ is the unique nonzero semigroup homomorphism from $S_{n}$ to $\{0,1\}$. Since $0 \in S_{n}, \xi$ is an isolated point of $\widehat{E(S)}$. Therefore, every Boolean action of $S_{n}$ has at most one fixed point, which becomes an isolated point.

\section{Acknowledgements}

The author would like to thank Professor Takeshi Katsura for his support and encouragement. The author is also grateful to the referees for useful comments. The author is also grateful to Tomoki Uchimura and Takaya Deto for giving the author comments about Proposition 5.1. 


\section{References}

[1] F. Komura, 'Quotients of étale groupoids and the abelianizations of groupoid C*-algebras', J. Aust. Math. Soc., to appear. Published online (7 April 2020).

[2] S. M. LaLonde and D. Milan, 'Amenability and uniqueness for groupoids associated with inverse semigroups', Semigroup Forum 95(2) (2017), 321-344.

[3] M. V. Lawson, Inverse Semigroups (World Scientific, Singapore, 1998).

[4] A. Paterson, Groupoids, Inverse Semigroups, and their Operator Algebras, Progress in Mathematics, 170 (Birkhäuser, Boston, MA, 2012).

[5] M. Petrich, Inverse Semigroups, Pure and Applied Mathematics (Wiley, New York, 1984).

[6] B. Piochi, 'Solvability in inverse semigroups', Semigroup Forum 34(1) (1986), 287-303.

[7] J. Renault, A Groupoid Approach to $C^{*}$-Algebras, Lecture Notes in Mathematics, 793 (Springer, Berlin, 1980).

[8] A. Sims, 'Hausdorff étale groupoids and their $\mathrm{C}^{*}$-algebras', in: Operator Algebras and Dynamics: Groupoids, Crossed Products, and Rokhlin Dimension, Advanced Courses in Mathematics, CRM Barcelona (ed. F. Perera) (Birkhäuser, Cham, 2020).

[9] B. Steinberg, 'A groupoid approach to discrete inverse semigroup algebras', Adv. Math. 223(2) (2010), 689-727.

FUYUTA KOMURA, Department of Mathematics,

Faculty of Science and Technology, Keio University, 3-14-1 Hiyoshi,

Kohoku-ku, Yokohama, 223-8522, Japan

e-mail: fuyuta.k@keio.jp 